\title{
Clinical Modality of Resistance and Subsequent Management of Patients with Advanced Non-small Cell Lung Cancer Failing Treatment with Osimertinib
}

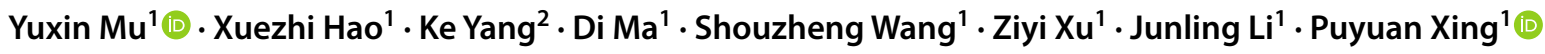

Published online: 23 May 2019

(c) The Author(s) 2019

\begin{abstract}
Background The third-generation epidermal growth factor receptor (EGFR)-tyrosine kinase inhibitor (TKI) osimertinib has become the standard treatment for patients with pretreated EGFR-mutated non-small cell lung cancer (NSCLC) who acquire the T790M resistance mutation. However, no standard treatment after osimertinib failure has been established.

Objective This study was undertaken to explore the clinical resistance modality upon failure of osimertinib therapy and to assess post-progression treatments in a real-world setting.

Patients and Methods Medical data were retrospectively collected in our cancer center of patients with advanced NSCLC treated between 1 March 2017 and 1 July 2018, and who developed resistance to osimertinib.

Results A total of 65 patients were analyzed. Clinical resistance modality varied among patients: 15 (23.1\%) with local progression, 29 (44.6\%) with gradual progression, and 21 (32.3\%) with dramatic progression. Most patients experienced intrathoracic progression only $(40 / 65,61.5 \%)$, while ten (15.4\%) cases presented intracranial failure only. Upon progressive disease, 20 patients (30.8\%) received subsequent chemotherapy, and showed a trend for longer median overall survival (OS) than in those receiving a non-chemotherapy regimen ( 25.0 vs. 11.8 months, $p=0.106)$. Thirty-nine patients $(60.0 \%)$ continued osimertinib beyond progression with a median post-progression treatment duration of 4.1 months. No significant difference in median OS was seen between patients who continued osimertinib and those who discontinued osimertinib (18.9 vs. 15.1 months, $p=0.802$ ). In subgroup analyses, OS was improved in patients who experienced dramatic progression and were treated with chemotherapy, but data were immature for patients with local or gradual progression.

Conclusions Chemotherapy could be an effective option after osimertinib failure in unselected patients.
\end{abstract}

Junling Li and Puyuan Xing contributed equally to this work.

Junling Li

lijunling@cicams.ac.cn

$\triangle$ Puyuan Xing

xingpuyuan@cicams.ac.cn

1 Department of Medical Oncology, National Cancer Center/ National Clinical Research Center for Cancer/Cancer Hospital, Chinese Academy of Medical Sciences and Peking Union Medical College, Number 17 Panjiayuan Nan Li, Chao Yang District, Beijing, China

2 Department of Medical Oncology, Cancer Hospital of HuanXing, ChaoYang District, Beijing, China

\section{Key Points}

In this real-world study, clinical modality of osimertinib resistance in patients with pretreated advanced NSCLC was mainly intrathoracic failure.

For patients experiencing progression on osimertinib, subsequent chemotherapy showed a trend for prolonged survival in a non-selected population, suggesting that chemotherapy is an effective post-resistance treatment option.

Continued osimertinib beyond progressive disease provided additional clinical benefit, but showed no overall survival benefit compared with patients who discontinued osimertinib in the non-selected population. 


\section{Introduction}

Non-small cell lung cancer (NSCLC) accounts for approximately $80 \%$ of all lung cancer cases [1]. Approximately $50 \%$ of Asian patients with NSCLC harbor activating epidermal growth factor receptor $(E G F R)$ mutations [2, 3]. In $E G F R$-mutant lung adenocarcinoma, targeted therapy with EGFR-tyrosine kinase inhibitors (TKIs) performs substantially better than standard cytotoxic chemotherapy in terms of efficacy and tolerability [4-7].

Osimertinib is an oral irreversible third-generation EGFR-TKI, which targets both activating mutations and the T790M resistance mutation, while sparing wild-type EGFR [8]. Several clinical trials have shown higher response rates (60-70\%) and longer progression-free survival (PFS) for osimertinib than platinum-based chemotherapy for advanced NSCLC patients with acquired T790M-mediated resistance to prior early-generation EGFR-TKIs [9]. However, despite initial benefits, progression inevitably occurs after about 10 months [9-11].

Efficacy of continued EGFR inhibition, cytotoxic chemotherapy, and combination therapy were reported after progression on early-generation EGFR-TKIs in the pre-osimertinib era [12-14]. Such clinical management options are all also used in patients who develop resistance to osimertinib, but data are lacking and clinical benefits are unclear. There is currently no established standard treatment for patients resistant to osimertinib. Hence, exploration of optimal therapy once osimertinib has failed is an area of highly unmet medical need. In this study, we assessed disease course and therapeutic options in patients with advanced NSCLC after progression on osimertinib in a real-world setting.

\section{Materials and Methods}

\subsection{Study Population}

Patients who received osimertinib between 1 March 2017 and 1 July 2018 were retrospectively analyzed in our cancer center. Inclusion criteria included histologically or cytologically confirmed NSCLC, advanced stage (including stage IIIB and IV), harboring the T790M mutation, receiving osimertinib as a second-line or later therapy, and developing progressive disease (PD) while on osimertinib treatment. Patients who received osimertinib for less than 3 weeks for any causes were excluded.

The study was approved by the Ethics Committee of the National Cancer Center/Cancer Hospital, Chinese Academy of Medical Sciences and Peking Union Medical College (approval no. 18-019).

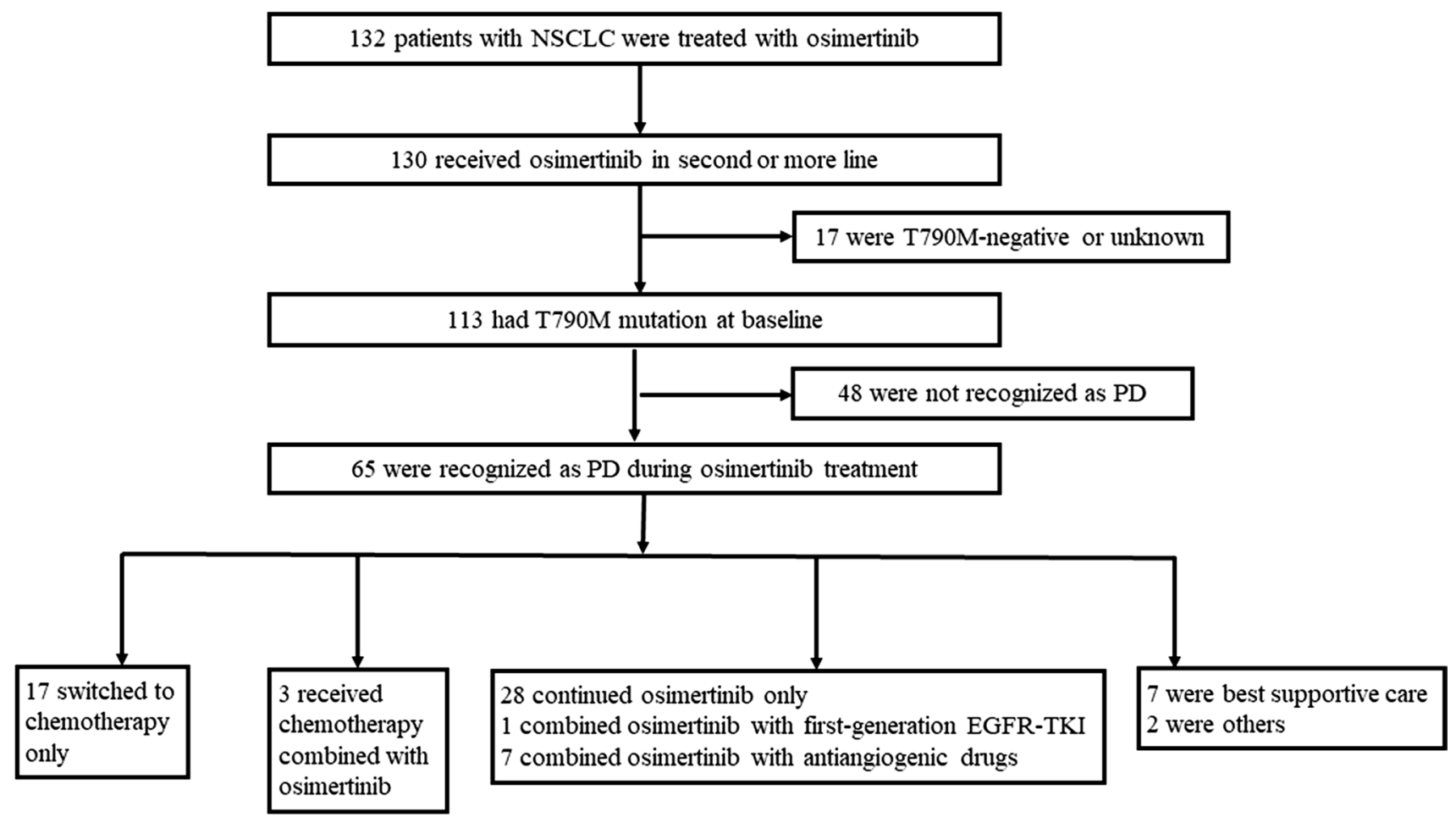

Fig. 1 Flowchart of patient selection. EGFR-TKI epidermal growth factor receptor-tyrosine kinase inhibitor, NSCLC non-small cell lung cancer, $P D$ progressive disease 
A flowchart for the selection of 65 patients who met all criteria and were included in this study is presented in Fig. 1. Data cutoff for follow-up was 31 March 2019.

\subsection{Assessments}

Our aim was to investigate the clinical modality of osimertinib therapy failure and evaluate the clinical benefit of subsequent therapeutic approaches in patients with advanced NSCLC. Tumor response was radiographically assessed according to Response Evaluation Criteria in Solid Tumors (RECIST) version 1.1. Upon disease progression, one pneumologist reviewed all patients and divided the patients into three groups, local progression, gradual progression, and dramatic progression, based on the evaluation of tumor burden on the computed tomography (CT) scan and clinical symptoms. Local progression was defined as new sites or locoregional regrowth with no more than three lesions in a limited area; gradual progression was defined as incremental increase in tumor burden through multiple sequential radiographic evaluations (radiologic assessment was undertaken about every 2 months according to medical records); and dramatic progression was defined as a rapid increase in tumor burden in a short period, or associated with obvious cancer-related symptoms.

Clinical activity endpoints of subsequent management included OS, PFS, objective response rate (ORR; complete or partial response), and disease control rate (DCR; stable disease, or complete or partial response). OS was defined as the time from the start of osimertinib to death from any cause, and post-progression overall survival (pOS) was defined from the date of PD on osimertinib until death from any cause. PFS was defined as the period between the start of osimertinib and first documentation of PD by RECIST version 1.1.

\subsection{Statistics Analysis}

ORR and DCR were compared using Chi square tests and Fisher's exact tests. OS and pOS were calculated using the Kaplan-Meier method and compared among groups using the log-rank test. Univariate and multivariate Cox regression analyses were used to explore potential prognostic factors for OS and pOS. The variables for multivariate analysis included sex, age, smoking status, Eastern Cooperative Oncology Group performance status (ECOG PS), central nervous system (CNS) metastasis, continuous osimertinib, chemotherapy post-PD, and dramatic progression. All reported $p$ values were two-sided and $p$ values $<0.05$ were considered to indicate statistical significance. All statistical analyses were performed using SPSS ${ }^{\circledR}$ version 23.0 statistical software (SPSS, Inc., Chicago, IL, USA).

\section{Results}

\subsection{Patients and Characteristics}

Sixty-five patients who developed resistance to osimertinib were analyzed. For the overall population, the median age was 59 years (range 39-86 years), 27 (41.5\%) patients were male, $16(24.6 \%)$ had a history of smoking, $62(95.4 \%)$ had a ECOG PS of 0 or 1, and 64 (98.5\%) had adenocarcinoma as confirmed by the initial biopsy specimen obtained before osimertinib therapy. Thirty-nine patients $(60.0 \%)$ received osimertinib as second-line therapy, 26 patients $(40.0 \%)$

Table 1 Baseline patient demographic and clinical characteristics

\begin{tabular}{|c|c|c|}
\hline \multirow[t]{2}{*}{ Characteristics } & \multicolumn{2}{|c|}{ Patients $(n=65)$} \\
\hline & $n^{\mathrm{a}}$ & $\%$ \\
\hline \multicolumn{3}{|l|}{ Age (years) } \\
\hline Median & 59 & \\
\hline Range & $39-86$ & \\
\hline \multicolumn{3}{|l|}{ Sex } \\
\hline Male & 27 & 41.5 \\
\hline Female & 38 & 58.5 \\
\hline \multicolumn{3}{|l|}{ ECOG PS } \\
\hline 0 & 33 & 50.8 \\
\hline 1 & 29 & 44.6 \\
\hline 2 & 3 & 4.6 \\
\hline \multicolumn{3}{|l|}{ Smoking status } \\
\hline Non-smoker & 49 & 75.4 \\
\hline Former/current smoker & 16 & 24.6 \\
\hline \multicolumn{3}{|l|}{ Histology } \\
\hline Adenocarcinoma & 64 & 98.5 \\
\hline Others & 1 & 1.5 \\
\hline \multicolumn{3}{|l|}{ Genotypes } \\
\hline \multicolumn{3}{|l|}{ T790M-positive } \\
\hline Exon 19del-positive & 27 & 41.5 \\
\hline L858R-positive & 34 & 52.3 \\
\hline Exon 19del/L858R-negative & 4 & 6.2 \\
\hline \multicolumn{3}{|l|}{ Osimertinib treatment line } \\
\hline 2 nd & 39 & 60.0 \\
\hline$\geq 3 \mathrm{rd}$ & 26 & 40.0 \\
\hline \multicolumn{3}{|l|}{ Metastases } \\
\hline Lung & 46 & 70.8 \\
\hline Bone & 33 & 50.8 \\
\hline CNS & 30 & 46.2 \\
\hline Pleural & 27 & 41.5 \\
\hline Liver & 11 & 16.9 \\
\hline Adrenal gland & 10 & 15.4 \\
\hline
\end{tabular}

CNS central nervous system, ECOG PS Eastern Cooperative Oncology Group performance status

${ }^{\mathrm{a}}$ Unless otherwise stated 
as third- or later-line therapy, and CNS metastases were detected in $30(46.2 \%)$ patients at baseline.

Before initiating osimertinib, most patients $(63 / 65$, 96.9\%) had received an early-generation EGFR-TKI; the other two patients had received chemotherapy. The median treatment duration (TD) of prior early-generation EGFRTKIs was 14.4 months (95\% confidence interval [CI] 11.5-17.3), and almost half of the patients experienced intrathoracic PD (31/63, 49.2\%). The median duration of follow-up from initial osimertinib treatment was 13.2 months. The PFS for osimertinib in the study population $(n=65)$ was 6.5 months (median; 95\% CI 5.1-7.9). Baseline demographic and treatment data are listed in Table 1.

\subsection{Clinical Modality of Resistance to Osimertinib and Subsequent Treatment}

The clinical modality of resistance varied among patients: 15 (23.1\%) experienced local progression, 29 $(44.6 \%)$ gradual progression, and $21(32.3 \%)$ dramatic progression. For patients with dramatic progression, the median OS and pOS was significantly inferior to that of patients with local or gradual progression (median OS, 8.3 [95\% CI 6.2-10.4] vs. 25.0 months [95\% CI 21.2-28.8], $p<0.001$; median pOS, 1.7 [95\% CI 0.7-2.7] vs. 18.1 months [95\% CI not reached $\{\mathrm{NR}\}], p<0.001$ ). As for the site of progression, most patients experienced intrathoracic progression only $(40 / 65,61.5 \%)$, while only ten $(15.4 \%)$ cases presented with intracranial failure only.

After evidence of PD, 39 of 65 patients $(60.0 \%)$ continued osimertinib. The median TD of post-progression osimertinib in this subgroup was 4.1 months (95\% CI 2.5-5.7), including 28 patients who received osimertinib ( 80 or $160 \mathrm{mg}$ once daily) alone, seven who received osimertinib plus an antiangiogenic drug, three with osimertinib plus chemotherapy, and one with osimertinib combined with a first-generation EGFR-TKI, with or without local treatment (including radiotherapy, intrathecal injection, and radiofrequency ablation). All of the four patients who received osimertinib $160 \mathrm{mg}$ daily postPD had experienced intracranial progression. Twentysix patients $(40.0 \%)$ discontinued osimertinib, among whom 17 switched to chemotherapy. The chemotherapy regimens were mostly platinum-based doublets $(13 / 20$, $65.0 \%$ ); other regimens included monotherapy pemetrexed and nab-paclitaxel with or without bevacizumab.

Most patients in the local or gradual progression subgroup continued osimertinib beyond PD (29/44, 65.9\%), which was a higher rate than in patients with dramatic progression $(10 / 21,47.6 \%)$, and the median TD of osimertinib post-progression in the local or gradual progression subgroup was 6.9 months (95\% CI 0.7-13.0). All of the ten patients who presented with intracranial failure alone continued osimertinib after resistance.

\subsection{Efficacy and Survival Outcomes}

Assessment of survival outcomes with post-progression therapy revealed that, on the one hand, there was a trend for both OS and pOS to be longer in patients who received chemotherapy than in those who did not receive chemotherapy after progression (median OS, 25.0 [95\% CI 18.1-31.9] vs. 11.8 months [95\% CI 3.4-20.2], $p=0.106$; median $\mathrm{pOS}$, 18.1 [95\% CI 4.4-31.8] vs. 6.9 months [95\% CI 0.0-14.6], $p=0.054)$. On the other hand, there was no statistically significant difference in OS and pOS between patients who continued osimertinib and patients who discontinued osimertinib after PD (median OS, 18.9 [95\% CI 8.5-29.3] vs. 15.1 months [95\% CI 4.1-26.1], $p=0.802$; median pOS, 13.5 [95\% CI 2.7-24.3] vs. 8.1 months [95\% CI 5.6-10.6], $p=0.832$ ). Also, there was no significant difference in patients who received chemotherapy and those who received a non-chemotherapy regimen after PD in terms of PFS and ECOG PS at baseline. ECOG PS was better at baseline in

Table 2 Cox regression for overall survival

\begin{tabular}{|c|c|c|c|c|}
\hline \multirow[t]{2}{*}{ Characteristics } & \multicolumn{2}{|l|}{ Univariate } & \multicolumn{2}{|l|}{ Multivariate } \\
\hline & HR $(95 \%$ CI $)$ & $p$ value & HR $(95 \%$ CI $)$ & $p$ value \\
\hline Male & $1.699(0.864-3.340)$ & 0.124 & $1.160(0.465-2.893)$ & 0.750 \\
\hline Age group ( $\leq 65$ years) & $0.640(0.315-1.300)$ & 0.217 & $1.049(0.446-2.464)$ & 0.913 \\
\hline Smoker & $1.325(0.613-2.868)$ & 0.474 & $1.139(0.419-3.095)$ & 0.798 \\
\hline $\mathrm{ECOG} P S=0$ & $0.403(0.196-0.828)$ & 0.013 & $0.464(0.208-1.037)$ & 0.061 \\
\hline CNS metastases & $1.092(0.551-2.165)$ & 0.800 & $1.778(0.789-4.004)$ & 0.165 \\
\hline Continued osimertinib & $0.914(0.461-1.811)$ & 0.796 & $0.510(0.190-1.372)$ & 0.182 \\
\hline Chemotherapy post-PD & $0.535(0.248-1.156)$ & 0.112 & $0.265(0.097-0.722)$ & 0.009 \\
\hline Dramatic PD & $4.579(2.289-9.161)$ & 0.000 & $4.126(1.865-9.129)$ & 0.000 \\
\hline
\end{tabular}

$C I$ confidence interval, $C N S$ central nervous system, ECOG PS Eastern Cooperative Oncology Group performance status, $H R$ hazard ratio, $P D$ progressive disease 
Table 3 Cox regression for post-progression overall survival

\begin{tabular}{|c|c|c|c|c|}
\hline \multirow[t]{2}{*}{ Characteristics } & \multicolumn{2}{|l|}{ Univariate } & \multicolumn{2}{|l|}{ Multivariate } \\
\hline & HR $(95 \%$ CI $)$ & $p$ value & HR $(95 \%$ CI $)$ & $p$ value \\
\hline Male & $1.365(0.702-2.652)$ & 0.359 & $0.955(0.361-2.529)$ & 0.927 \\
\hline Age group ( $\leq 65$ years $)$ & $0.604(0.298-1.225)$ & 0.162 & $1.000(0.426-2.348)$ & 0.999 \\
\hline Smoker & $1.054(0.492-2.259)$ & 0.893 & $1.441(0.525-3.955)$ & 0.478 \\
\hline $\mathrm{ECOG} P S=0$ & $0.379(0.186-0.774)$ & 0.008 & $0.352(0.158-0.784)$ & 0.011 \\
\hline CNS metastases & $1.005(0.512-1.972)$ & 0.989 & $1.330(0.624-2.837)$ & 0.460 \\
\hline Continued osimertinib & $0.926(0.467-1.835)$ & 0.825 & $0.437(0.162-1.178)$ & 0.102 \\
\hline Chemotherapy post-PD & $0.469(0.212-1.039)$ & 0.062 & $0.124(0.040-0.383)$ & 0.000 \\
\hline Dramatic PD & $4.638(2.348-9.159)$ & 0.000 & 7.607 (3.242-17.850) & 0.000 \\
\hline
\end{tabular}

$C I$ confidence interval, $C N S$ central nervous system, ECOG PS Eastern Cooperative Oncology Group performance status, $H R$ hazard ratio, $P D$ progressive disease

\section{(A) OS in patients with or without chemotherapy}

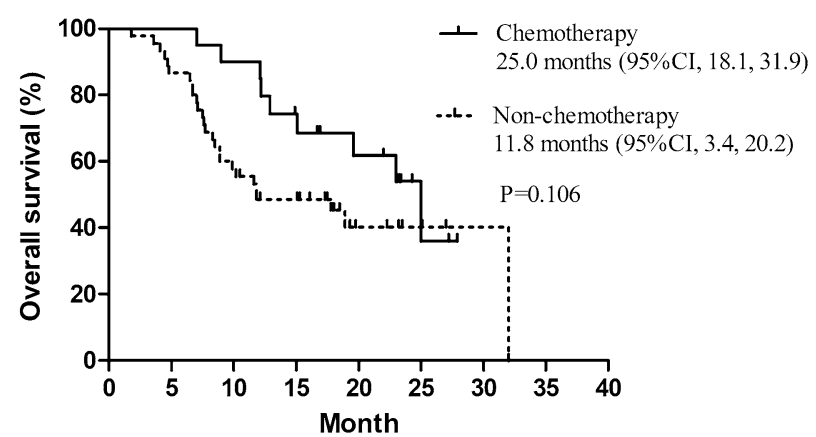

(C) pOS in patients with or without chemotherapy

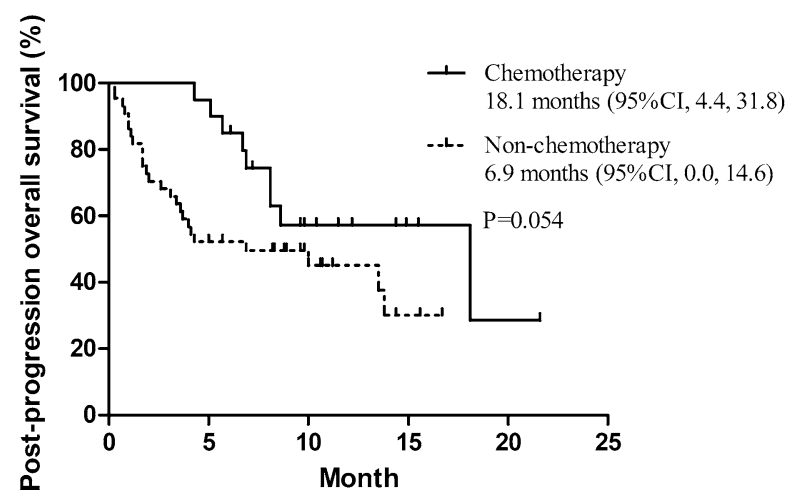

Fig. 2 Overall survival in patients with or without chemotherapy after progression on osimertinib (a) and in patients who continued or discontinued osimertinib after progression (b), and post-progression overall survival in patients with or without chemotherapy after

patients with continued osimertinib post-PD, compared with those who discontinued, but no difference in terms of PFS between the two groups was observed.
(B) OS in patients continued or discontinued osimertinib

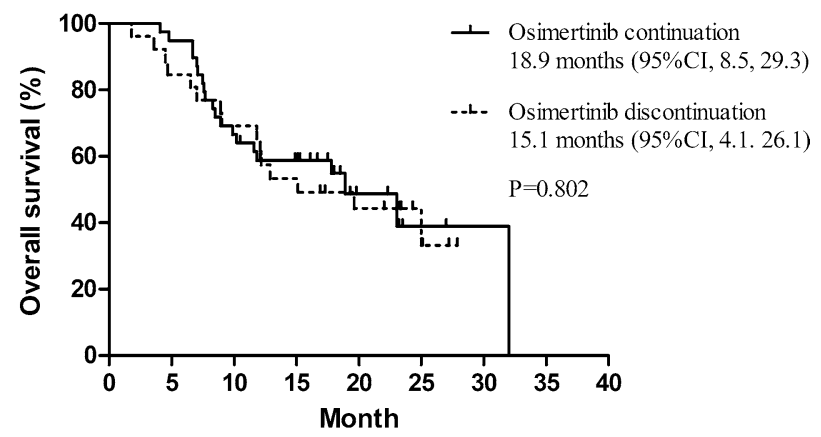

(D) pOS in patients continued or discontinued osimertinib

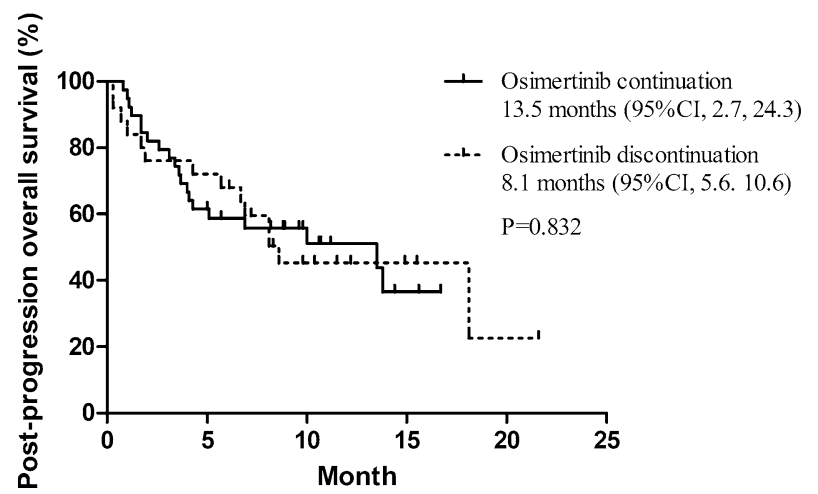

progression on osimertinib (c) and in patients who continued or discontinued osimertinib after progression (d). Tick marks indicate censored events. $C I$ confidence interval, $O S$ overall survival, $p O S$ postprogression overall survival

In the subgroup of patients with dramatic progression, OS and pOS were significantly longer in patients who received chemotherapy than in the non-chemotherapy regimen (median OS, 12.9 [95\% CI NR] vs. 7.5 months [95\% 
CI 6.0-9.0], $p=0.006$; median pOS, 8.1 [95\% CI NR] vs. 1.1 months [95\% CI 0.7-1.5], $p<0.001)$. OS and pOS were immature in the subgroup of patients with local or gradual progression. Results for the Cox regression analyses are listed in Tables 2 and 3. Chemotherapy post-PD contributed significantly to longer OS and pOS in the multivariate analysis. A survival benefit was also observed in the local or gradual progression group compared with the dramatic progression population. Results of the survival analysis are shown in Fig. 2.

After resistance to osimertinib, 45 patients were evaluable for response analysis of post-progression treatment. The ORR was higher, although not significantly, in patients with than in patients without chemotherapy (25.0\% vs. $3.4 \%$, $p=0.088)$. There was no difference in DCR for patients with chemotherapy and those without $(75.0 \%$ vs. $75.9 \%$, $p=1.000)$. No statistically significant improvement in the osimertinib continuation group in terms of ORR and DCR was found compared with patients who discontinued osimertinib (ORR, $6.9 \%$ vs. $18.8 \%, p=0.474$; DCR, $75.9 \%$ vs. $75.0 \%, p=1.000)$

\subsection{Translational Assessment}

Of 65 patients, 30 with paired molecular information for both pre-osimertinib and resistance reassessment were available for molecular analysis. The molecular information was gathered using next-generation sequencing (NGS) from plasma or tissue samples. T790M loss was the most common phenomenon observed in 17 patients $(17 / 30,56.7 \%)$, among whom eight patients $(8 / 17,47.1 \%)$ continued osimertinib beyond PD and showed a median TD post-progression of 3.3 months (95\% CI 0.2-6.4). Thirteen patients retained the T790M mutation $(13 / 30,43.3 \%)$ at the time of resistance, among whom ten patients $(10 / 13,76.9 \%)$ continued osimertinib with a median TD post-progression of 6.0 months (95\% CI 0.0-14.6). Patients who retained the T790M mutation showed a trend for longer TD post-progression than in patients with T790M loss (median 6.0 vs. 3.3 months, $p=0.811$ ) when osimertinib was used beyond PD. OS and pOS were not mature, as only 4 of 13 and 6 of 17 patients had died in the T790M-retained and T790M-loss populations, respectively, at the time of data cutoff.

\section{Discussion}

To improve the understanding of the clinical modality of resistance to osimertinib and to investigate survival outcomes of post-resistance treatments, we retrospectively assessed the medical records of 65 osimertinib-resistant NSCLC patients. The data revealed that subsequent chemotherapy was a key prognostic factor for longer survival and should be considered in patients developing PD to osimertinib.

Our data indicate that the diversity of the clinical modality of osimertinib failure could help inform strategies for subsequent treatment and predict prognosis. Median OS and pOS were significantly longer in patients with local or gradual progression than in those with dramatic progression. Switching to chemotherapy improved survival compared with other therapeutic regimens in the dramatic progression population. For the local or gradual progression population, OS and pOS data were not mature in our study, but similar data have been reported after resistance to early-generation EGFR-TKIs in the pre-osimertinib era [15]. Previous data demonstrated a phenomenon of rapid clinical progression after discontinuation of EGFR-TKIs [16, 17]; thus, inhibition of the EGFR pathway beyond progression was explored in early-generation EGFR-TKIs. In the phase II, single-arm ASPIRATION (Asian Pacific trial of Tarceva as first-line in EGFR mutation) study, the median OS in patients who continued erlotinib beyond PD was 33.6 (95\% CI 27.3-34.3) months compared with 22.5 (95\% CI 20.1-27.0) months for patients who did not continue erlotinib treatment [13]. In IMPRESS (Iressa Mutation-Positive Multicentre Treatment Beyond ProgRESsion Study), a randomized phase III study, patients who continued gefitinib plus chemotherapy presented an OS improvement compared with patients who received chemotherapy only after progression on first-line gefitinib. The LUX-Lung 7 study showed a median TD beyond progression of 2.7 months for afatinib and 2.0 months for gefitinib [18]. Other available evidence was mostly from small retrospective studies [19-21]. Yu et al. [22] reported an impressive OS of 41 months and PFS of 10 months in 18 NSCLC patients who received local treatment with continued TKI after oligometastatic PD on EGFR-TKIs. Although the results were mostly from retrospective studies, and may be biased by investigators' choice, continuation of EGFR-TKIs with or without local treatment has become an option for patients with local or slow progression in the pre-osimertinib era.

Continued osimertinib treatment beyond progression similarly resulted in a median post-progression TD of 4.1 months in our study, providing additional clinical benefit, but showed no OS benefit compared with patients who did not continue in the overall non-selected population.

Subsequent chemotherapy after PD, however, resulted in a survival benefit in non-selected patients in our study. Longer previous exposure to EGFR inhibitors, the presence of more heterogenous tumors, and more complex molecular mechanisms of acquired resistance to osimertinib compared with early-generation EGFR-TKIs may help explain why survival was more favorable with chemotherapy in our study, especially for the subgroup of patients with dramatic progression. 
Intrathoracic failure, rather than isolated CNS progression, was more frequently seen on osimertinib, which may further explain the significance of chemotherapy after osimertinib progression. CNS metastases affect $30-50 \%$ of NSCLC patients throughout the course of the disease [23], which has been partly blamed on the inferior blood-brain barrier (BBB) penetration by early-generation EGFRTKIs. Locoregional CNS failure would not have been considered to be due to acquired systemic resistance to early-generation EGFR-TKI therapy, and continuous TKI administration beyond the occurrence of CNS PD was indicated. As BBB penetration of osimertinib is improved over early-generation EGFR-TKIs, in our study only ten (15.4\%) cases presented with intracranial failure alone. Chemotherapy may perform better once osimertinib failure has occurred, considering the reasons mentioned earlier in this section. Le et al. [24] recently reported a study on osimertinib continuation beyond progression in EGFRmutant NSCLC. The study showed that continuation of osimertinib beyond PD was associated with a longer OS than was discontinuation (11.2 vs. 6.1 months, $p=0.02)$, which was different to our study. However, for patients receiving additional treatment without osimertinib, only eight of 24 (33.3\%) patients received chemotherapy in this study compared with 17 of $26(65.4 \%)$ in our study. The difference in post-progression treatments between the studies may have resulted in different outcomes.

Various resistance mechanisms to osimertinib and subsequent treatments have been reported. T790M loss and T790M retention were two basic modes, which may inform strategies for subsequent treatment and predict survival. Our study showed a longer osimertinib TD postprogression in patients who retained the T790M mutation than in those who presented T790M loss, which means T790M-retained tumors may benefit more from osimertinib continuation treatment. Unfortunately, reassessment of molecular information in our study was performed in limited cases, and median OS was not reached. Further studies exploring molecular resistance information-based treatment selection are needed.

Additional EGFR mutations, such as the C797S mutation, were the most common mechanisms of resistance to osimertinib, and combining EAI045 or afatinib with cetuximab could be used to treat such patients [25, 26]. The combination of chemotherapy, bevacizumab, and atezolizumab in the IMpower (Immunology power) 150 study showed a median PFS of 9.7 months in an EGFR-mutated population who have failed to respond to a prior EGFR-TKI [27]. Other treatment strategies such as combining chemotherapy and pembrolizumab in the Keynote 789 trial are also ongoing. Although additional new treatment strategies are emerging, new medications or inclusion in clinical trials may not be possible or available for all patients. Chemotherapy could still be an option, especially in chemotherapy-naïve patients and when the mechanism of resistance is unknown. A study assessed the efficacy of platinum-doublet chemotherapy in NSCLC patients harboring EGFR mutations as second-line treatment and demonstrated that PFS and ORR were not different between T790M-positive and T790M-negative populations [28]. The study indicated that chemotherapy might be a feasible option after osimertinib failure, regardless of the T790M status at the time of resistance.

Our study was limited by its single-center, retrospective design, and relatively small sample size of patients with post-osimertinib molecular tests. The heterogeneity of treatment lines and subsequent treatments and the overlap of patients who continued osimertinib and who received chemotherapy may have biased the survival results of the different post-osimertinib treatment approaches in our study. In addition, few patients in our study were re-biopsied; thus, potential histologic transformation may have influenced survival outcomes.

\section{Conclusion}

Once osimertinib resistance has occurred in pretreated NSCLC patients, re-biopsy and reassessment of molecular information is recommended to guide subsequent treatment options. In cases where the mechanism of resistance is unknown or new medications or inclusion in clinical trials are not available, chemotherapy is a feasible option and can lead to prolonged survival in the non-selected population. Continued osimertinib beyond PD in the local or gradual progression population and T790M-retained patients may provide additional clinical benefit, and further study is needed regarding whether combination of continued osimertinib with chemotherapy provides further survival benefit.

Acknowledgements The authors would like to thank all study patients and their family members.

\section{Compliance with Ethical Standards}

Funding No external funding was used in the preparation of this manuscript.

Conflict of interest Yuxin Mu, Xuezhi Hao, Ke Yang, Di Ma, Shouzheng Wang, Ziyi Xu, Junling Li, and Puyuan Xing declare that they have no conflicts of interest that might be relevant to the contents of this manuscript.

Open Access This article is distributed under the terms of the Creative Commons Attribution-NonCommercial 4.0 International License (http://creativecommons.org/licenses/by-nc/4.0/), which permits any noncommercial use, distribution, and reproduction in any medium, provided you give appropriate credit to the original author(s) and the 
source, provide a link to the Creative Commons license, and indicate if changes were made.

\section{References}

1. Miller KD, Siegel RL, Lin CC, Mariotto AB, Kramer JL, Rowland $\mathrm{JH}$, et al. Cancer treatment and survivorship statistics, 2016. CA Cancer J Clin. 2016;66(4):271-89.

2. Midha A, Dearden S, McCormack R. EGFR mutation incidence in non-small-cell lung cancer of adenocarcinoma histology: a systematic review and global map by ethnicity (mutMapII). Am J Cancer Res. 2015;5(9):2892-911.

3. Shi Y, Au JS, Thongprasert S, Srinivasan S, Tsai CM, Khoa MT, et al. A prospective, molecular epidemiology study of EGFR mutations in Asian patients with advanced non-small-cell lung cancer of adenocarcinoma histology (PIONEER). J Thorac Oncol. 2014;9(2):154-62.

4. Ohashi K, Maruvka YE, Michor F, Pao W. Epidermal growth factor receptor tyrosine kinase inhibitor-resistant disease. J Clin Oncol. 2013;31(8):1070-80.

5. Mok TS, Wu YL, Thongprasert S, Yang CH, Chu DT, Saijo N, et al. Gefitinib or carboplatin-paclitaxel in pulmonary adenocarcinoma. N Engl J Med. 2009;361(10):947-57.

6. Rosell R, Carcereny E, Gervais R, Vergnenegre A, Massuti B, Felip E, et al. Erlotinib versus standard chemotherapy as first-line treatment for European patients with advanced EGFR mutation-positive non-small-cell lung cancer (EURTAC): a multicentre, open-label, randomised phase 3 trial. Lancet Oncol. 2012;13(3):239-46.

7. Sequist LV, Yang JC, Yamamoto N, O’Byrne K, Hirsh V, Mok $\mathrm{T}$, et al. Phase III study of afatinib or cisplatin plus pemetrexed in patients with metastatic lung adenocarcinoma with EGFR mutations. J Clin Oncol. 2013;31(27):3327-34.

8. Cross DA, Ashton SE, Ghiorghiu S, Eberlein C, Nebhan CA, Spitzler PJ, et al. AZD9291, an irreversible EGFR TKI, overcomes T790M-mediated resistance to EGFR inhibitors in lung cancer. Cancer Discov. 2014;4(9):1046-61.

9. Mok TS, Wu Y, Ahn M, Garassino MC, Kim HR, Ramalingam SS, et al. Osimertinib or platinum-pemetrexed in EGFR T790Mpositive lung cancer. N Engl J Med. 2017;376(7):629-40.

10. Goss G, Tsai CM, Shepherd FA, Bazhenova L, Lee JS, Chang GC, et al. Osimertinib for pretreated EGFR Thr790 Met-positive advanced non-small-cell lung cancer (AURA2): a multicentre, open-label, single-arm, phase 2 study. Lancet Oncol. 2016;17(12):1643-52.

11. Yang JC, Ahn MJ, Kim DW, Ramalingam SS, Sequist LV, Su WC, et al. Osimertinib in pretreated T790M-positive advanced non-small-cell lung cancer: AURA study phase II extension component. J Clin Oncol. 2017;35(12):1288-96.

12. Mok T, Kim SW, Wu YL, Nakagawa K, Yang JJ, Ahn MJ, et al. Gefitinib plus chemotherapy versus chemotherapy in epidermal growth factor receptor mutation-positive non-small-cell lung cancer resistant to first-line gefitinib (IMPRESS): overall survival and biomarker analyses. J Clin Oncol. 2017;35(36):4027-34.

13. Park K, Yu CJ, Kim SW, Lin MC, Sriuranpong V, Tsai CM, et al. First-line erlotinib therapy until and beyond response evaluation criteria in solid tumors progression in Asian patients with epidermal growth factor receptor mutation-positive non-small-cell lung cancer: the ASPIRATION study. JAMA Oncol. 2016;2(3):305-12.

14. Goldberg SB, Oxnard GR, Digumarthy S, Muzikansky A, Jackman DM, Lennes IT, et al. Chemotherapy with erlotinib or chemotherapy alone in advanced non-small cell lung cancer with acquired resistance to EGFR tyrosine kinase inhibitors. Oncologist. 2013;18(11):1214-20.
15. Yang JJ, Chen HJ, Yan HH, Zhang XC, Zhou Q, Su J, et al. Clinical modes of EGFR tyrosine kinase inhibitor failure and subsequent management in advanced non-small cell lung cancer. Lung Cancer. 2013;79(1):33-9.

16. Riely GJ, Kris MG, Zhao B, Akhurst T, Milton DT, Moore E, et al. Prospective assessment of discontinuation and reinitiation of erlotinib or gefitinib in patients with acquired resistance to erlotinib or gefitinib followed by the addition of everolimus. Clin Cancer Res. 2007;13(17):5150-5.

17. Chaft JE, Oxnard GR, Sima CS, Kris MG, Miller VA, Riely GJ. Disease flare after tyrosine kinase inhibitor discontinuation in patients with EGFR-mutant lung cancer and acquired resistance to erlotinib or gefitinib: implications for clinical trial design. Clin Cancer Res. 2011;17(19):6298-303.

18. Park K, Tan EH, O’Byrne K, Zhang L, Boyer M, Mok T, et al. Afatinib versus gefitinib as first-line treatment of patients with EGFR mutation-positive non-small-cell lung cancer (LUX-Lung 7): a phase $2 \mathrm{~B}$, open-label, randomised controlled trial. Lancet Oncol. 2016;17(5):577-89.

19. Asami K, Okuma T, Hirashima T, Kawahara M, Atagi S, Kawaguchi $\mathrm{T}$, et al. Continued treatment with gefitinib beyond progressive disease benefits patients with activating EGFR mutations. Lung Cancer. 2013;79(3):276-82.

20. Nishie K, Kawaguchi T, Tamiya A, Mimori T, Takeuchi N, Matsuda $\mathrm{Y}$, et al. Epidermal growth factor receptor tyrosine kinase inhibitors beyond progressive disease: a retrospective analysis for Japanese patients with activating EGFR mutations. J Thorac Oncol. 2012;7(11):1722-7.

21. Faehling M, Eckert R, Kamp T, Kuom S, Griese U, Strater J, et al. EGFR-tyrosine kinase inhibitor treatment beyond progression in long-term Caucasian responders to erlotinib in advanced nonsmall cell lung cancer: a case-control study of overall survival. Lung Cancer. 2013;80(3):306-12.

22. Yu HA, Sima CS, Huang J, Solomon SB, Rimner A, Paik P, et al. Local therapy with continued EGFR tyrosine kinase inhibitor therapy as a treatment strategy in EGFR-mutant advanced lung cancers that have developed acquired resistance to EGFR tyrosine kinase inhibitors. J Thorac Oncol. 2013;8(3):346-51.

23. Bhatt VR, Kedia S, Kessinger A, Ganti AK. Brain metastasis in patients with non-small-cell lung cancer and epidermal growth factor receptor mutations. J Clin Oncol. 2013;31(25):3162-4.

24. Le X, Puri S, Negrao MV, Nilsson MB, Robichaux J, Boyle T, et al. Landscape of EGFR-dependent and -independent resistance mechanisms to osimertinib and continuation therapy beyond progression in EGFR-mutant NSCLC. Clin Cancer Res. 2018;24(24):6195-203.

25. Jia Y, Yun CH, Park E, Ercan D, Manuia M, Juarez J, et al. Overcoming EGFR (T790M) and EGFR (C797S) resistance with mutant-selective allosteric inhibitors. Nature. 2016;534(7605):129-32.

26. Janjigian YY, Smit EF, Groen HJ, Horn L, Gettinger S, Camidge DR, et al. Dual inhibition of EGFR with afatinib and cetuximab in kinase inhibitor-resistant EGFR-mutant lung cancer with and without T790M mutations. Cancer Discov. 2014;4(9):1036-45.

27. Reck M, Mok T, Nishio M, Jotte RM, Cappuzzo F, Orlandi F, et al. Atezolizumab plus bevacizumab and chemotherapy in non-small-cell lung cancer (IMpower150): key subgroup analyses of patients with EGFR mutations or baseline liver metastases in a randomised, open-label phase 3 trial. Lancet Respir Med. 2019;7(5):387-40125. https://doi.org/10.1016/S2213 -2600(19)30084-0.

28. Yoshida T, Kuroda H, Oya Y, Shimizu J, Horio Y, Sakao Y, et al. Clinical outcomes of platinum-based chemotherapy according to T790M mutation status in EGFR-positive non-small cell lung cancer patients after initial EGFR-TKI failure. Lung Cancer. 2017;109:89-91. 